\title{
Benefit-Risk Analysis of Upadacitinib Compared with Adalimumab in the Treatment of Patients with Moderate-to-Severe Rheumatoid Arthritis
}

\author{
Philip Conaghan · Stanley Cohen · Gerd Burmester • Eduardo Mysler • \\ Peter Nash · Yoshiya Tanaka · William Rigby · Jayeshkumar Patel • \\ Tim Shaw $\cdot$ Keith A. Betts · Pankaj Patel · Jianzhong Liu • \\ Rochelle Sun · Roy Fleischmann
}

Received: October 4, 2021 / Accepted: November 8, 2021 / Published online: November 23, 2021

(C) The Author(s) 2021

\section{ABSTRACT}

Introduction: Rheumatoid arthritis (RA) is a chronic autoimmune disease requiring longterm treatment. Upadacitinib (UPA), a Janus kinase (JAK) inhibitor, is a new treatment for RA. The benefit-risk profile of a medication is best understood by evaluating the number needed to treat (NNT) and the number needed

Supplementary Information The online version contains supplementary material available at https:// doi.org/10.1007/s40744-021-00399-5.

\section{P. Conaghan}

Leeds Institute of Rheumatic and Musculoskeletal Medicine, National Institute for Health Research, Leeds Biomedical Research Centre-University of Leeds, Leeds, UK

S. Cohen

Metroplex Clinical Research Center, Dallas, TX, USA

G. Burmester

Charité-Universitätsmedizin Berlin, Berlin, Germany

E. Mysler

Organización Médica de Investigación, Buenos

Aires, Argentina

P. Nash

Griffith University, Brisbane, Queensland, Australia

Y. Tanaka

The First Department of Internal Medicine, University of Occupational and Environmental

Health Japan, Kitakyushu, Japan to harm $(\mathrm{NNH})$. This analysis evaluated the comparative risk-benefit of UPA versus adalimumab (ADA).

Methods: Post-hoc analyses were performed using data from the SELECT-COMPARE trial of UPA versus placebo (PBO) and UPA versus ADA among patients with active RA who remained on stable methotrexate (MTX) treatment and had an inadequate response; patients who failed to achieve response were rescued by predefined criteria-PBO or ADA switch to UPA, and UPA switch to ADA (all patients on PBO were switched to UPA at week 26). This analysis assessed efficacy

\section{W. Rigby}

Dartmouth-Hitchcock Medical Center, Lebanon, $\mathrm{NH}$, USA

J. Patel $(\varangle) \cdot$ P. Patel · J. Liu

AbbVie, Inc, $26525 \mathrm{~N}$ Riverwoods Blvd, Mettawa,

North Chicago, IL 60045, USA

e-mail: jayesh.patel@abbvie.com

T. Shaw

AbbVie Ltd, Maidenhead, UK

K. A. Betts

Analysis Group, Inc, Los Angeles, CA, USA

R. Sun

Analysis Group, Inc, Boston, MA, USA

R. Fleischmann

Metroplex Clinical Research Center, University of Texas Southwestern Medical Center, Dallas, TX, USA 
and adverse events of special interest (AESIs) at week 26, 48, and 156 (3 years). NNT and NNH (95\% confidence intervals) values were calculated between UPA versus ADA for all time points, and between UPA versus PBO for week 26 . NNT and NNH values were applied to a hypothetical cohort of 100 patients to estimate the comparative efficacy and safety profiles.

Results: UPA consistently showed greater efficacy than ADA, as evidenced by NNT values $<10$ for achievement of Disease Activity Score in 28 joints based on C-reactive protein (DAS28-CRP) of $<2.6$ and $\leq 3.2$, respectively, and functional improvement. Based on indices for disease assessment other than the DAS28-CRP, remission outcomes were higher with UPA versus ADA over 26 weeks (NNTs: 7-12), 48 weeks (NNTs: 9-16), and 156 weeks (NNTs: 9-15). With the exception of herpes zoster, other AESIs demonstrated a similar risk with UPA versus ADA.

Conclusion: In patients with active RA despite MTX use, UPA demonstrated an incremental achievement of clinical outcomes compared to ADA together with a similar profile of AESIs with ADA (with the exception of herpes zoster).

Keywords: Benefit-risk assessment; Number needed to treat; Number needed to harm; Efficacy; Safety; Rheumatoid arthritis; Upadacitinib; Janus kinase inhibitor

\section{Key Summary Points}

Why carry out this study?

Rheumatoid arthritis (RA) is a chronic and highly debilitating disease that can lead to irreversible joint damage, loss of physical function, and reduced quality of life if not properly treated.

There are patients who experience an inadequate response or intolerance to treatment with methotrexate (MTX) and/ or to second-line treatment with biologic therapies, such as the tumor necrosis factor alpha inhibitor, adalimumab (ADA); inhibitors of Janus kinase, such as upadacitinib (UPA), could help to address this unmet need.
Utilizing data from the phase III randomized, double-blind SELECTCOMPARE trial, this analysis employed a number needed to treat and number needed to harm methodology to evaluate the benefit and risk of UPA versus ADA at weeks 26,48 , and 156 among patients with active RA despite continued therapy with MTX.

\section{What was learned from the study?}

UPA showed greater efficacy compared to ADA for the achievement of meaningful clinical and functional improvements at 26,48 , and 156 weeks, with a similar safety profile to ADA (with the exception of an additional safety risk of herpes zoster when compared to ADA).

When conducting a benefit-risk assessment among a hypothetical cohort of 100 patients, the present analysis estimated a greater number of patients who would experience benefit rather than harm from UPA treatment in comparison with ADA at 26, 48, and 156 weeks.

Given the favorable benefit-risk profile of UPA compared to ADA in the present study, UPA could be considered as an effective and generally safe treatment option for patients with active RA receiving background MTX.

\section{INTRODUCTION}

Rheumatoid arthritis (RA) is a chronic, inflammatory, autoimmune disease that requires longterm treatment. If not properly treated, patients with RA may sustain irreversible joint damage, resulting in reduced physical function $[1,2]$, disability [3], work productivity loss [4, 5], and reduced quality of life $[2,6]$. Thus, the control of inflammation is critical for reducing the burden of disease and represents a primary goal of RA treatment [2, 7-9].

For patients who experience an inadequate response or intolerance to first-line 
conventional synthetic disease-modifying antirheumatic drugs (csDMARDs), current guidelines recommend biologic disease-modifying antirheumatic drugs (bDMARDs) as second-line treatment [10]. bDMARDs approved for the treatment of RA include tumor necrosis factor alpha (TNF- $\alpha$ ) inhibitors, interleukin 6 inhibitors, T-cell signal inhibitors, and anti-D D 20 antibodies, all of which have substantially improved RA disease management and control $[11,12]$. However, despite this progress, less than half of patients receiving biologic therapy achieve low disease activity or remission with stringent metrics outcomes that are associated with better economic, clinical, and humanistic outcomes [13-15].

More recently, inhibitors of Janus kinase (JAK) have emerged as a promising new class of targeted therapy for RA $[16,17]$. By inhibiting various JAK isoforms (notably JAK1, but also JAK2 and JAK3), these novel agents partially block the activity of multiple pro-inflammatory cytokines simultaneously, thereby reducing the uncontrolled inflammatory and immune responses seen in RA $[16,17]$. Unlike biologics, which have long half-lives and require injections or infusions, JAK inhibitors are small molecule drugs, have a short half-life, are nonimmunogenic, and can be taken orally [17]. However, as relatively new treatments, evidence of their safety continues to emerge through randomized clinical trials and realworld evidence. Some studies have indicated a number of potential safety signals, including an elevated risk of herpes zoster and possibly an elevated risk of venous thromboembolic (VTE) events, major cardiovascular events (MACE), and malignancies [18-20]. Placing these potential risks in the context of the potential benefits associated with JAK inhibitors is important to better understand their benefit-risk profile, especially relative to more established treatments, such as TNF- $\alpha$ inhibitors.

Upadacitinib (UPA) is a selective and reversible JAK inhibitor that preferentially inhibits signaling by JAK1 or JAK1/3 with functional selectivity over cytokine receptors that signal via pairs of JAK2 [21, 22]. Worldwide regulatory agencies have approved UPA $15 \mathrm{mg}$ once daily, taken orally, for use in patients with moderateto-severe RA after inadequate response or intolerance to a course of methotrexate (MTX) [23-25].

UPA has demonstrated efficacy and safety in five double-blind, randomized controlled phase III trials [26-30]. One of these trials, SELECTCOMPARE, included a direct head-to-head comparison of UPA and a TNF- $\alpha$ inhibitor, adalimumab (ADA). At 12 weeks, treatment with UPA was associated with superior rates of ACR50 ( $\geq 50 \%$ improvement in American College of Rheumatology score), improvement in pain, and improvement in physical function, as well as significantly higher proportions of patients achieving remission by multiple metrics [30]. The improvements over ADA were maintained for up to 3 years [31]. Although not powered for safety, the study also indicated that, with the exception of a higher rate of herpes zoster and elevated creatine phosphokinase (CPK), liver enzymes, and lipids in patients receiving UPA, the safety profiles of UPA and ADA were generally comparable.

Direct head-to-head comparisons of new and existing treatments in randomized controlled trials, such as SELECT-COMPARE, provide the gold standard for comparative evidence. By utilizing a number needed to treat (NNT) and number needed to harm (NNH) methodology, such studies can provide an understanding of the incremental benefits and risks of new treatments relative to an established treatment. Evaluated together, NNT and NNH are valuable effect size metrics that can offer useful comparisons of clinically relevant benefits and harms, allowing physicians to make informed treatment decisions.

The objective of the present analysis was to employ NNT and NNH analyses to evaluate the efficacy and safety outcomes from the SELECTCOMPARE trial in order to quantify and contextualize the incremental benefit and risk of UPA compared to ADA in patients with RA [32]. 


\section{METHODS}

\section{Data Source and Study Design}

A post-hoc analysis was performed using data collected in SELECT-COMPARE (ClinicalTrials.gov: NCT02629159), a phase III, randomized, double-blind study comparing UPA to PBO and to ADA in patients with moderately to severely active RA who were on a stable background of MTX and who had an inadequate response to MTX. [30, 33] This multicenter trial was conducted at 286 sites in 41 countries and included a total of 1629 patients with active RA (based on ACR classification criteria and radiographic evidence) who had received MTX for $\geq 3$ months at a stable dosage of $15-25 \mathrm{mg} /$ week for $\geq 4$ weeks prior to the first dose of study drug. Included patients were randomized 2:2:1 to receive UPA $15 \mathrm{mg}$ once daily, $\mathrm{PBO}$, or ADA $40 \mathrm{mg}$ every other week in combination with a stable background dose of MTX. At weeks 14,18 , and 22, patients who did not experience minimum improvement of $\geq 20 \%$ in tender joint count and swollen joint count from baseline received blinded rescue therapy, which included switching from PBO to UPA, from UPA to ADA, or from ADA to UPA. At week 26, all patients randomized to $\mathrm{PBO}$ were switched to UPA treatment, irrespective of their response to prior treatment. Patients randomized to UPA or ADA who did not achieve low disease activity by the Clinical Disease Activity Index (CDAI $\leq 10)$, following the treat-to-target principle, were also switched to the alternative treatment [34]. Subsequently, patients continued treatment (UPA or ADA) in a blinded manner until the last patient completed the week 48 visit; patients continued to receive the same treatment in an open-label manner thereafter. Open-label follow-up visits occurred at weeks 60 and 72, and every 12 weeks thereafter. Data presented herein include results up to week 156 (3 years). A summary of trial design and patient disposition throughout the trial duration are shown in Electronic Supplementary Material (ESM) Figs. 1 and 2.

In the current post-hoc analysis, efficacy outcomes were analyzed using non-responder imputation among the full analysis set, which included all patients who received at least one dose of the assigned study drug. Trial results across all time points were analyzed by randomized group. For efficacy outcomes up to week 48 , patients who met the rescue criteria at weeks 14,18 , or 22 were considered as non-responders at visits after receiving rescue treatment. For patients who were rescued at week 26 , the last response prior to rescue was carried forward after treatment switching. For efficacy at week 156, all patients who were rescued, including those rescued at week 26 , were considered as non-responders after rescue treatment switching.

Adverse event counts were censored for patients who switched to and initiated rescue treatment or after a pre-specified observation period for each randomized treatment if the patient discontinued prematurely from the study. As such, for patients who met the rescue criteria prior to week 26 , events that occurred starting the day of initiation of rescue treatment were excluded for the initial study drug (i.e., PBO, UPA, or ADA). Safety data for weeks 48 and 156 were summarized among patients who remained on their randomized treatment (i.e., UPA or ADA) and therefore excluded observations among patients who switched treatment due to rescue. Specific criteria for inclusion in the trial population have been published previously [30].

The SELECT-COMPARE study was conducted according to the International Conference on Harmonisation guidelines, applicable regulations, and the Declaration of Helsinki. Studyrelated documents were approved by the U.S. Central Institutional Review Board (Quorum \#31,009) and other local institutional ethics committees and review boards. All patient data used in this analysis were de-identified and certified as fully compliant with U.S. patient confidentiality requirements outlined in the Health Insurance Portability and Accountability Act of 1996. Because this study did not involve the collection, use, or dissemination of individually-identifiable data, institutional review board approval was not necessary. 


\section{Efficacy and Safety Outcomes}

This post-hoc analysis focused on key efficacy and safety outcomes evaluated in the SELECTCOMPARE trial. Categorical efficacy outcomes included achievement of improved response, clinical remission, low disease activity, and functional improvement as defined by multiple indices for disease assessment. The achievement of clinical remission and low disease activity were assessed using several established definitions. In particular, one of the primary endpoints in the SELECT-COMPARE trial was the achievement of a Disease Activity Score in 28 joints based on C-reactive protein (DAS28-CRP) $<2.6$ [30]. Accordingly, DAS28-CRP results were assessed in the present study with those patients achieving a score of $<2.6$ and $\leq 3.2$ [35]. Additionally, the present study assessed scores on the CDAI, which defines remission as $\mathrm{CDAI} \leq 2.8$ and low disease activity as CDAI $\leq 10$, as well as the Simple Disease Activity Index (SDAI), which defines remission as SDAI $\leq 3.3$ and low disease activity as SDAI $\leq 11$ [36]. Boolean-based remission rates recommended by the ACR and European League Against Rheumatism (EULAR) were also evaluated [37]. Finally, the Health Assessment Questionnaire Disability Index (HAQ-DI) normative cutoff of $\leq 0.25$ was used to assess functional status in RA patients [38]. Clinically relevant adverse events (AEs) included any AEs, discontinuation due to AEs, serious AEs (SAEs), and AEs of special interest (AESIs), including serious infection, herpes zoster, malignancies (excluding nonmelanoma skin cancer [NMSC]), major adverse cardiovascular events ((VTEs) MACE, and thromboembolic VTE events. Detailed descriptions of safety and efficacy outcomes are provided in ESM Table 1.

The evaluated efficacy and safety outcomes were selected as they represent what are considered the most clinically relevant endpoints to inform the benefit and risk of UPA $[35,37]$.

\section{Statistical Analyses}

NNT and NNH were calculated by taking the reciprocal of the response rate or risk difference, respectively, between the observed rate for UPA compared with PBO (up to week 26) or compared with ADA (for weeks 26, 48, and 156) for each outcome of interest. The $95 \%$ confidence intervals (95\% CIs) for NNT and NNH were calculated by taking the reciprocals of values defining the confidence interval of the response rate difference for each outcome [39]. In cases where the range of the confidence intervals for NNT or NNH included infinity, the comparison was determined to be not significant, and is represented by 'ns.'

NNT can be interpreted as the estimated number of patients that would need to be treated with the active treatment (e.g., UPA) to achieve one additional efficacy outcome of interest versus the comparator (e.g., $\mathrm{PBO}$ or ADA) [40, 41]. Similarly, NNH represents the estimated number of patients that would need to be treated before observing one additional safety outcome of interest versus the comparator [42]. As such, lower NNT values are favorable for efficacy outcomes, suggesting that efficacy benefits can be observed by treating a marginal number of patients with the therapy of interest. In contrast, higher $\mathrm{NNH}$ values are favorable when evaluating safety outcomes, as they indicate that a higher number of patients would need to be treated before observing an additional adverse event. Conversely, if the treatment of interest demonstrates even lower incidence of an adverse event relative to the comparator, resulting $\mathrm{NNH}$ values would be negative, suggesting that additional AEs are less likely to occur when a number of patients are treated with the therapy of interest [43]. Evaluated together, NNT and NNH are valuable effect size metrics that can offer useful comparisons of clinically relevant benefits and harms.

The NNT and NNH values were applied to a hypothetical cohort of 100 patients to jointly evaluate the estimated number of additional efficacy and safety outcomes that would be observed if these 100 patients were treated with UPA instead of ADA. The cohort of patients is assumed to share the average demographic and clinical characteristics of patients evaluated in the SELECT-COMPARE trial [30]. The estimated incremental number of patients achieving efficacy outcomes and AESIs per 100 patients 


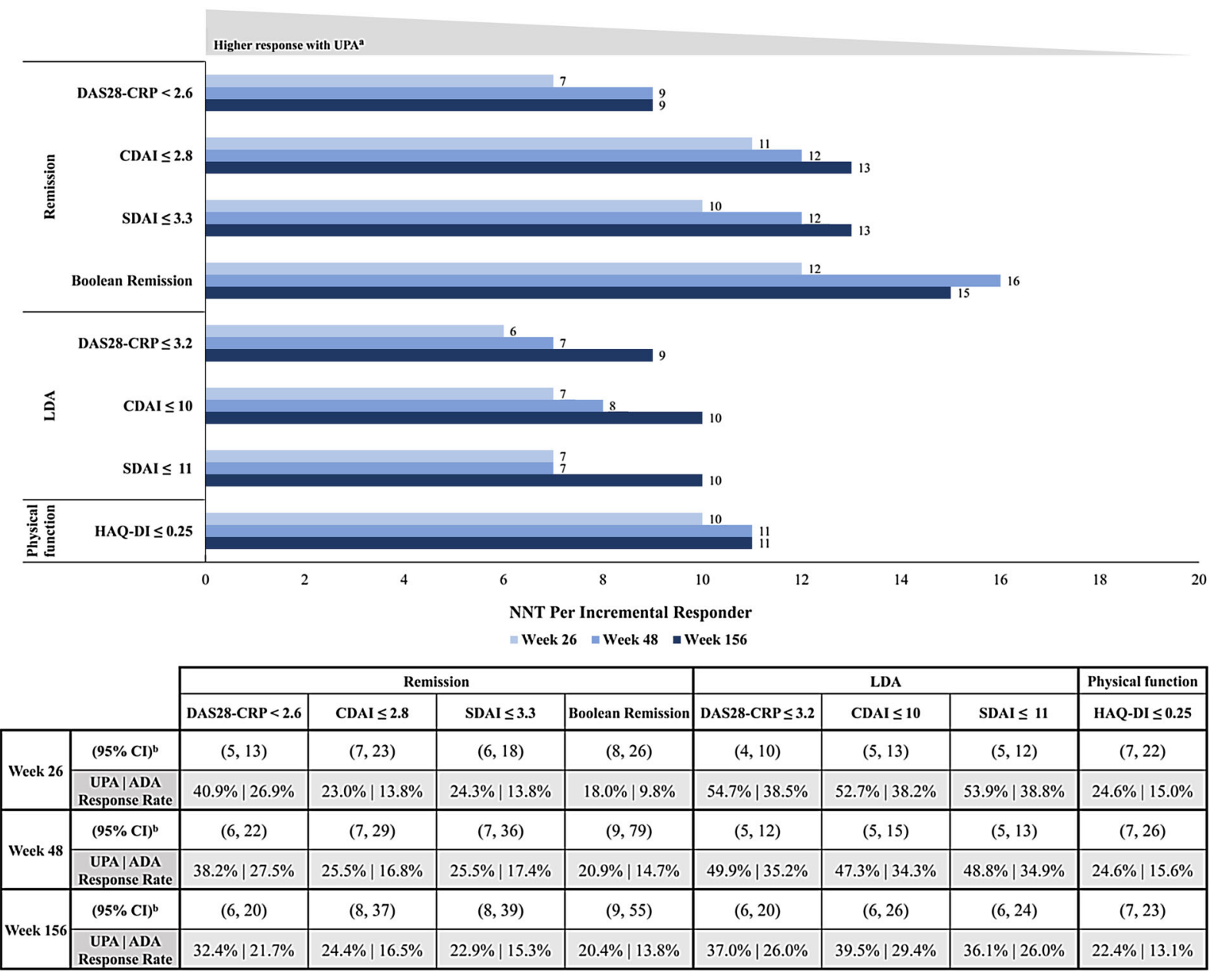

Fig. 1 Number needed to treat per incremental responder-UPA vs. ADA. Superscript a: Positive values approaching 0 increasingly favor UPA treatment over ADA. Superscript b: $95 \%$ CIs for NNH/NNT estimates calculated by taking reciprocals of the values defining the confidence interval for the response rate difference. $A D A$

receiving UPA was calculated by dividing 100 by the NNT and NNH values.

\section{RESULTS}

\section{NNT Results}

The calculated NNT values (95\% CIs) at 26, 48, and 156 weeks are presented in Fig. 1 . The response rate difference for achieving DAS28$\mathrm{CRP}<2.6$ between UPA and ADA at 26 weeks
Adalimumab, $C D A I$ Clinical Disease Activity Index, $C I$ confidence interval, DAS28-CRP Disease Activity Score in 28 joints using C-reactive protein, HAQ-DI Health Assessment Questionnaire Disability Index, $L D A$ low disease activity, $N N T$ number-needed-to-treat, SDAI Simple Disease Activity Index, UPA upadacitinib

was $13.9 \%$, resulting in an NNT of 7 (95\% CI: 5, 13). In other words, for every seven patients treated with UPA instead of ADA for 26 weeks, it is estimated that one additional patient would achieve DAS28-CRP $<2$.6. For CDAI-, SDAI- and Boolean-defined remission, NNT values $(95 \%$ CIs) of $11(7,23), 10(6,18)$, and $12(8,26)$ were observed, respectively.

Similar results were observed at 48 weeks; the NNT values $(95 \% \mathrm{CI})$ for efficacy outcomes were $9(6,22)$ for DAS28-CRP < 2.6, $12(7,29)$ for CDAI remission, $12(7,36)$ for SDAI remission, 
and $16(9,79)$ for Boolean remission. Among low disease activity outcomes at 26 and 48 weeks, the NNTs ranged from 6 to 8 (Fig. 1).

Patients receiving UPA continued to experience numerically higher remission rates compared to patients receiving ADA at week 156 (3 years), resulting in NNTs (95\% CIs) of 9 (6, 20) for DAS28-CRP $<2.6,13(8,37)$ for CDAI, $13(8,39)$ for SDAI, and $15(9,55)$ for the Boolean criteria. Among low disease activity outcomes, the NNTs ranged from 9 to 10 at 156 weeks.

NNT results comparing outcomes between UPA and PBO are presented in ESM Fig. 3. Relative efficacy benefits were greater in comparisons between UPA and PBO, resulting in NNT values ranging between 3 and 7 across all efficacy outcomes evaluated.

\section{NNH Results}

NNH values (95\% CIs) at 26,48 , and 156 weeks are presented in Fig. 2. At week 26, patients receiving UPA demonstrated a lower incidence of serious AEs, discontinuation due to AEs, MACE, and VTE compared to those receiving $\mathrm{ADA}$, resulting in negative $\mathrm{NNH}$ values. $\mathrm{NNH}$ values for serious AEs and discontinuation due to AEs were $-170(\mathrm{~ns})$ and -33 (95\% CI: -817 , - 17). As such, treatment of 170 patients over 26 weeks with UPA instead of ADA would result in one fewer SAE, while treatment of 33 patients would result in one fewer discontinuation due to $\mathrm{AE}(\mathrm{s})$. Adjudicated MACE was observed in zero $(0.0 \%)$ patients receiving UPA and in two $(0.6 \%)$ patients receiving ADA, resulting in an $\mathrm{NNH}$ of -164 (ns). Adjudicated VTE was observed in two $(0.3 \%)$ patients receiving UPA and in three $(0.9 \%)$ patients receiving $\mathrm{ADA}$, resulting in an $\mathrm{NNH}$ of -164 (ns). Among other outcomes, $4 \%$ more patients receiving UPA experienced any AEs, resulting in a NNH of 26 (ns). Serious infection and herpes zoster were observed in $12(1.8 \%)$ and five $(0.8 \%)$ patients receiving UPA, respectively, and in five $(1.5 \%)$ and one $(0.3 \%)$ patient receiving $\mathrm{ADA}$, respectively, resulting in a NNH of 315 (ns) for serious infection and 216 (ns) for herpes zoster. Relative differences between treatments were not statistically significant across AE outcomes with the exception of discontinuation due to AEs.

At 48 weeks, patients receiving UPA experienced lower risk for most AESIs and AE categories compared with patients receiving ADA. Rates of SAEs and discontinuation due to AEs resulted in negative NNH values of -17 (ns) and -20 (ns), respectively. Consistent with this, other AESIs were observed in a lower proportion of patients treated with UPA versus ADA, with NNH of - 39 (ns) for serious infection, - 106 (ns) for MACE, - 291 (ns) for malignancy other than NMSC, and - 56 (ns) for VTE. More patients (3.0\%) treated with UPA experienced any AEs, resulting in an $\mathrm{NNH}$ of 33 (ns), while $2.4 \%$ more patients treated with UPA experienced herpes zoster infection, resulting in an NNH of 41 (95\% CI: 22, 266).

Results were directionally similar at 156 weeks (3 years). Specifically, $6.6 \%$ and 5.5\% fewer patients treated with UPA versus ADA experienced SAEs or discontinuation due to AEs, resulting in $\mathrm{NNH}$ values of -15 (ns) and -18 (ns), respectively. Risk differences and $\mathrm{NNH}$ values for other AESIs generally favored UPA over ADA, with values of -34 (ns) for serious infection, - 106 (ns) for MACE, - 270 (ns) for malignancy other than NMSC, and - 78 (ns) for VTE. In total, $3.9 \%$ more patients treated with UPA experienced any AEs, resulting in an NNH of 26 (ns), and $3.7 \%$ more patients treated with UPA experienced herpes zoster infection, resulting in an NNH of 27 (95\% CI: 15, 136). NNH results comparing outcomes between UPA and $\mathrm{PBO}$ are presented in ESM Fig. 4.

\section{Benefit-Risk Profile}

A favorable benefit-risk profile was observed with UPA compared to ADA at 26, 48, and 156 weeks (Figs. 3, 4). Among a hypothetical cohort of 100 patients, treatment with UPA instead of ADA was estimated to lead to an additional 14 patients achieving DAS28-CRP < 2.6 at week 26 and to 11 patients achieving DAS28-CRP $<2.6$ at weeks 48 and 156. CDAI remission is expected to be achieved by nine additional patients at 26 and 48 weeks and by eight additional patients at 156 weeks, while 


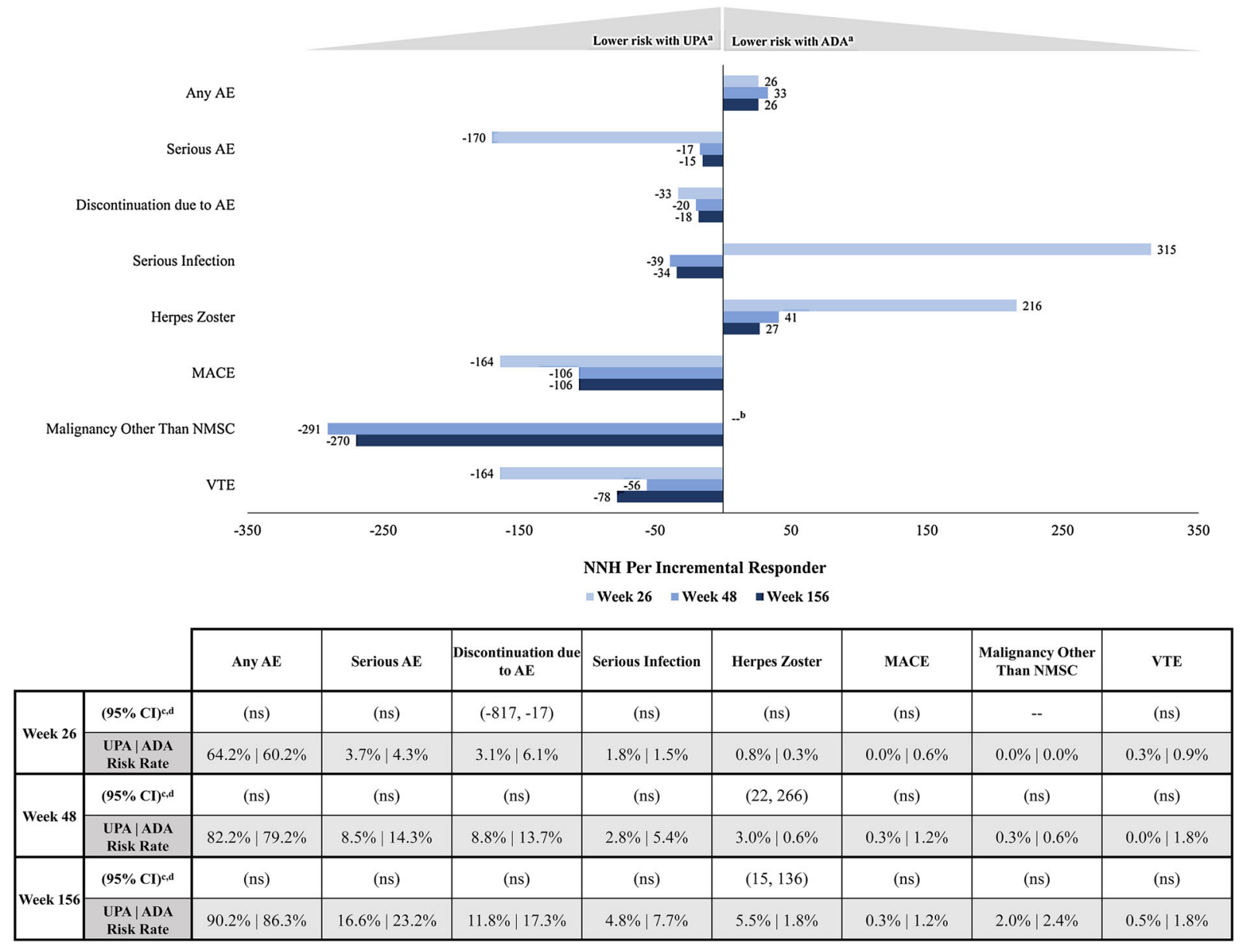

Fig. 2 Number needed to harm per incremental responder-UPA vs. ADA. Superscript a: Negative values approaching 0 increasingly favoring UPA treatment over ADA treatment, indicating a greater absolute difference in the number of events experienced between patients receiving UPA or ADA. Positive values approaching 0 increasingly favor ADA treatment over UPA. Superscript b: Zero malignancies other than NMSC were observed in either arm; therefore, NNH was not calculated. Superscript c: $95 \%$ CIs for NNH/NNT estimates were calculated by taking reciprocals of the values defining the

SDAI remission is expected to be achieved by 11 additional patients at 26 weeks and by eight additional patients at 48 and 156 weeks. At least ten additional patients are expected to achieve DAS28-CRP $\leq 3.2$, low disease activity according to CDAI, or low disease activity according to SDAI at all time points evaluated. For normative HAQ-DI results, ten additional patients are expected to achieve HAQ-DI $\leq 0.25$ at week 26 confidence interval for the response rate difference. Superscript d: Positive NNH values indicate the number of patients needed to treat with UPA instead of ADA to experience one additional adverse event, while negative $\mathrm{NNH}$ values indicate the number of patients needed to treat with UPA instead of ADA to experience one fewer adverse event. $A D A$ Adalimumab, $A E$ Adverse event, $C I$ Confidence Interval, $M A C E$ major adverse cardiovascular events, $N M S C$ non-melanoma skin cancer, $N N H$ numberneeded-to-harm, ns not significant, UPA Upadacitinib, $V T E$ venous thromboembolic events

and nine additional patients are expected to achieve the outcomes at 48 and 156 weeks.

The corresponding number of patients who would likely experience additional AEs over 26 weeks ranged from three fewer patients discontinuing due to AEs to four additional patients experiencing any AEs among the hypothetical cohort of 100 patients. Approximately one fewer serious AE, MACE, and VTE 


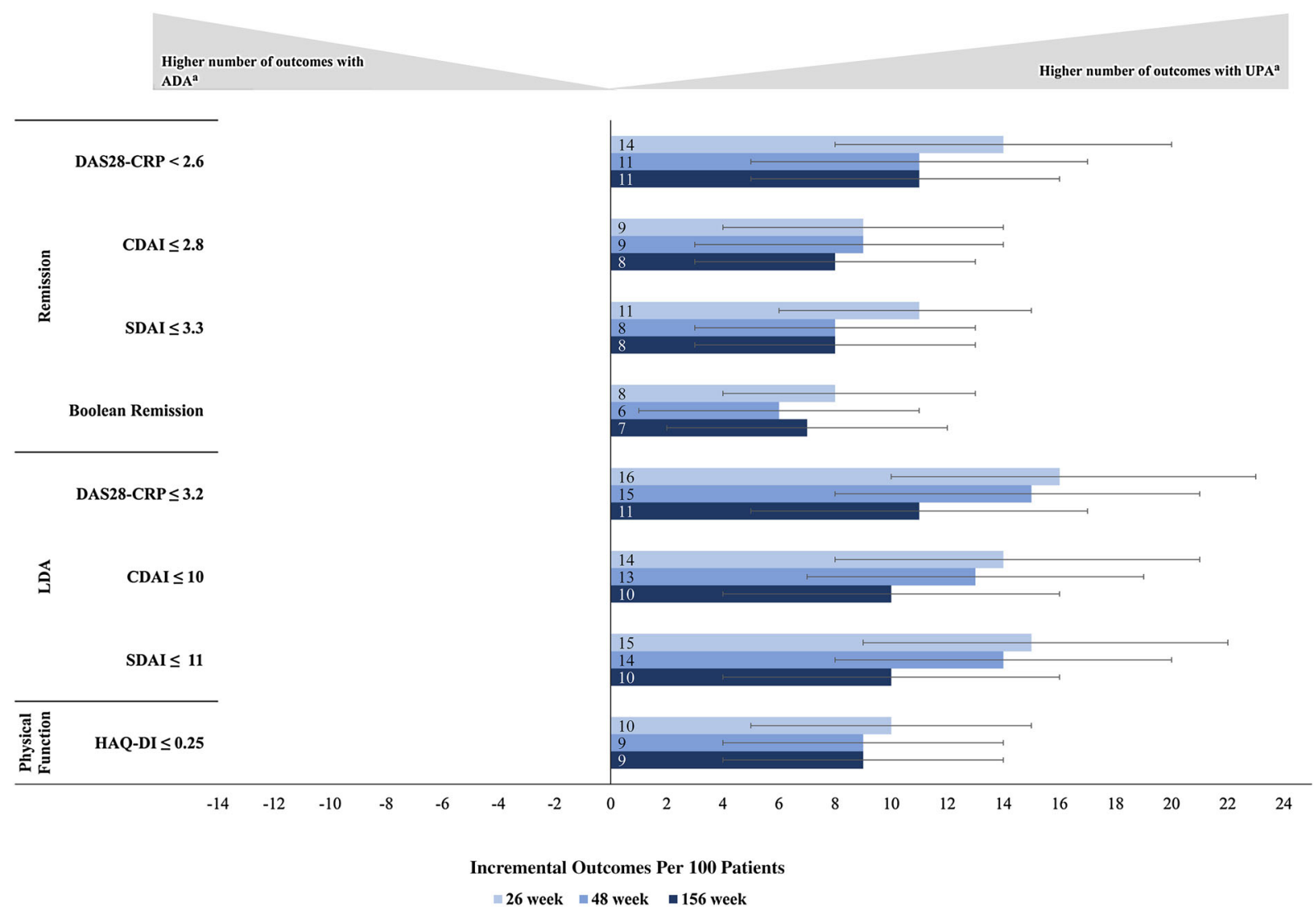

Fig. 3 Incremental number of patients achieving clinical benefit per 100 patients treated with UPA vs. ADA. Superscript a: Increasing positive values indicate that a higher number of clinical outcomes are expected to be observed in the cohort of 100 patients who receive UPA treatment instead of ADA. $A D A$ Adalimumab, $C D A I$

would be estimated to occur with the treatment of 100 patients with UPA instead of ADA for 26 weeks. Results are similar at later time points of evaluation. At 48 and 156 weeks, even fewer patients are expected to experience serious $\mathrm{AE}$, discontinuation due to AEs, and serious infection. Two additional herpes zoster infections would be estimated to occur at 48 weeks and four additional herpes zoster infections would be estimated after 156 weeks of treatment.

\section{DISCUSSION}

In the current post-hoc analysis based on data from the SELECT-COMPARE trial, the NNT and $\mathrm{NNH}$ values of UPA compared to those of ADA
Clinical Disease Activity, DAS-28-CRP Disease Activity Score in 28 joints using C-reactive protein, $H A Q-D I$ Health Assessment Questionnaire Disability Index, $L D A$ Low Disease Activity, SDAI Simple Disease Activity Index, $U P A$ Upadacitinib

indicate a favorable benefit-risk profile of UPA among patients with moderate-to-severely active RA who have inadequate response to MTX. While the absolute efficacy rates from the SELECT-COMPARE trial have been previously published, these NNT and NNH results provide an intuitive and practical interpretation of relative treatment performance for physicians to consider when guiding treatment decisions.

In the present analysis, the lower NNT values demonstrate that few additional patients would need to be treated with UPA versus ADA to have one additional patient achieve important RAspecific efficacy outcomes, including remission, low disease activity, and normal physical function. Most notably, the NNTs for UPA versus 


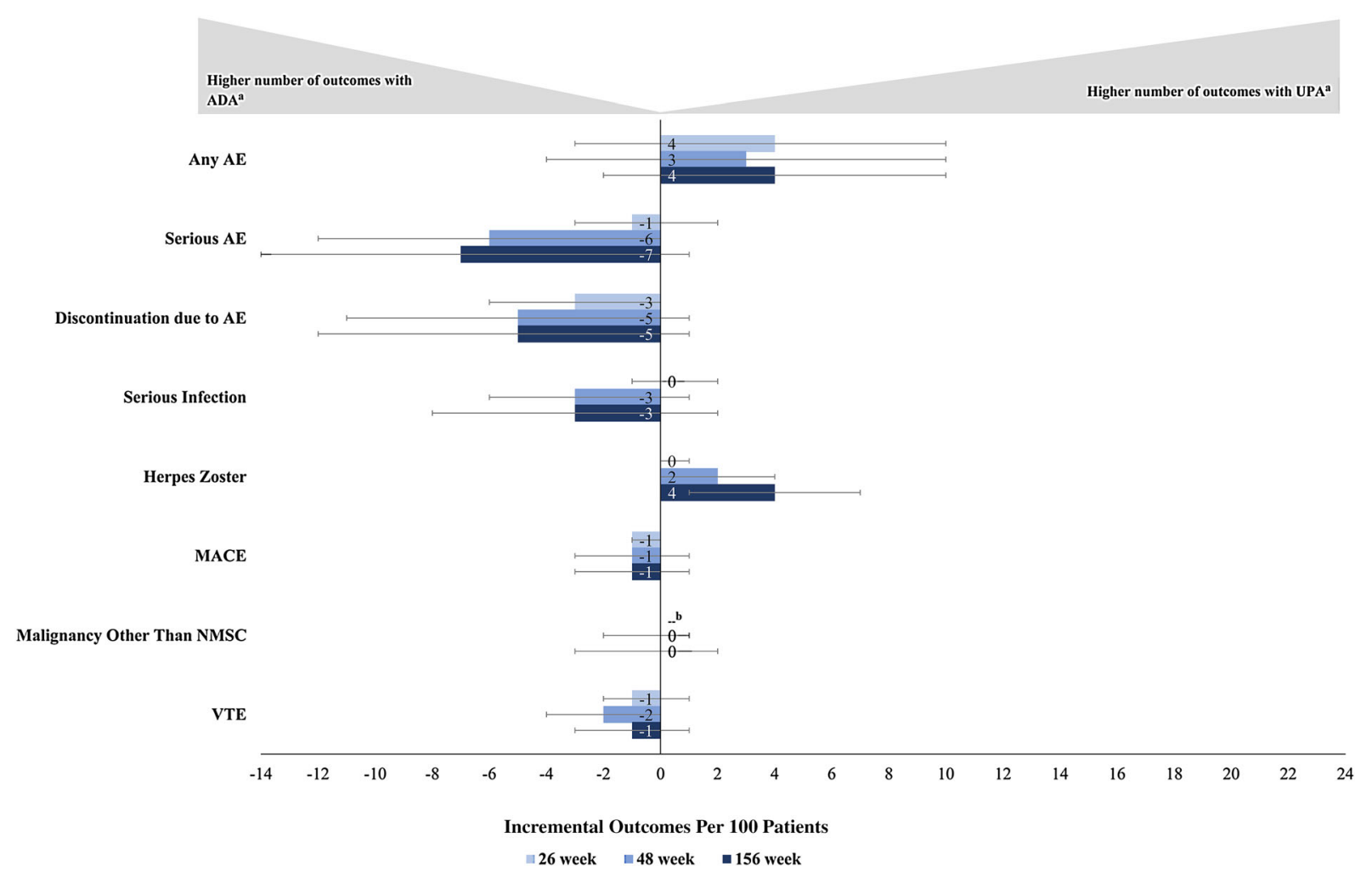

Fig. 4 Incremental number of patients experiencing adverse events per 100 patients treated with UPA versus ADA. Superscript a: Increasing positive values indicate that a higher number of events are expected to be observed in a cohort of 100 patients who receive UPA treatment instead of ADA. Negative values indicate that fewer events are expected to be observed in a cohort of 100 patients

ADA to achieve additional remission outcomes at 26 weeks ranged from 7 to 12 , and these were maintained through 156 weeks. The comparative effect sizes of UPA for these remission outcomes provide strong supportive evidence for the positive efficacy profile of UPA versus ADA, as these outcomes represent more stringent measures of disease control and have been found to be associated with positive health and disease outcomes [37]. Furthermore, this study demonstrates the benefit of treatment for patient-reported disability assessments, namely through the evaluation of patients achieving a normative HAQ-DI score of $\leq 0.25$. A normative HAQ-DI score is a more stringent cutoff compared to commonly used minimal clinically important differences to evaluate disability in who receive UPA treatment instead of ADA. Superscript b: Zero malignancies other than NMSC were observed in either arm; therefore, incremental outcomes were not calculated. $A D A$ Adalimumab, $A E$ Adverse Event, $M A C E$ Major Adverse Cardiovascular Events, NMSC Non-Melanoma Skin Cancer, UPA Upadacitinib, VTE Venous Thromboembolic Events

patients affected by RA and allows for comparisons of results across clinical populations [44].

By leveraging data from the SELECT-COMPARE trial, the results of the present analysis demonstrate a consistent benefit of UPA relative to ADA using comparative data collected in a controlled clinical trial setting. While the superior efficacy of an active investigational treatment versus a PBO control arm is traditionally the minimally required result to demonstrate therapeutic treatment benefit in patients, the superior efficacy of UPA versus the ADA active control arm highlights the added benefit that UPA provides over the current standard of care. The effect sizes of outcome differences between UPA and ADA indicated by the smaller NNT values reported across all 
evaluated dimensions demonstrate the value of UPA as an important treatment option to fill unmet needs observed among patients with RA who are intolerant to or experience an inadequate response to csDMARDs. Prior studies reporting NNT and NNH values among other JAK inhibitors have focused on comparisons with $\mathrm{PBO}$, which limits the interpretability of the treatment's value within the existing therapeutic landscape of RA $[45,46]$. In the SELECTCOMPARE trial, the PBO arm was associated with worse efficacy results than the ADA arm across all efficacy outcomes evaluated, and the resulting NNT values comparing UPA to $\mathrm{PBO}$ ranged from 3 to 7 at week 26 (ESM Fig. 3). These results further emphasize the benefit of considering alternative treatment options for patients who are intolerant to or who fail to achieve adequate response to MTX treatment.

The current study also assessed a variety of important safety outcomes to evaluate the risks associated with UPA treatment. These safety outcomes included SAEs as an aggregate category and key AESIs as highlighted in the product labels for UPA and other JAK inhibitors [23, 24, 47-50]. Negative NNH values for most AEs (including SAEs, serious infections, MACE, malignancies and VTEs) indicate that these AEs were more frequently observed in patients treated with $\mathrm{ADA}$, demonstrating lower risk for UPA. Additionally, the majority of NNHs (including negative NNHs) were associated with 95\% CIs that included infinity, which indicates that the differences between treatments were too small to be considered significant. The only AEs with a NNH and a 95\% CI not including infinity were AEs leading to discontinuation at week 26 (favoring UPA) and herpes zoster infection at weeks 48 and 156 (favoring ADA). However, no serious or severe herpes zoster infections were reported during the trial among patients who were randomized to UPA or ADA treatment. While some safety differences between UPA and ADA have been previously identified, including CPK elevations, hepatic disorders, and lymphopenia [30], this current analysis of AESIs remains consistent with earlier reports observing similar rates of these events with both UPA and ADA. It is worth noting that many of the evaluated AEs are rare events with low incidence. Therefore, the direct clinical applicability of mean $\mathrm{NNH}$ values should be interpreted with caution and evaluated alongside the absolute AE rates. When the benefit and risk were evaluated together in a hypothetical cohort of 100 patients, our study estimated a greater number of patients who would experience benefit rather than harm from UPA treatment in comparison with ADA treatment; these findings further support the positive benefit-risk profile of UPA relative to ADA.

All in all, NNT and NNH values are very context-dependent. As a general rule of thumb, smaller NNT values for important clinical outcomes (e.g., remission or low disease activity) and larger $\mathrm{NNH}$ values for relevant AEs (e.g., infection) are preferable. Relative comparisons between the efficacy outcomes and safety events evaluated in the benefit-risk analysis provides additional insight into the interpretation of NNT and NNH values.

The present study findings may have been subject to certain limitations inherent in benefit-risk assessments. First, a limitation of NNT and $\mathrm{NNH}$ measures is that they are limited to binary outcomes. Second, the applicability of NNT and NNH values and comparative risk-benefit results are also dependent on the response rates observed in the comparator arm within the trial populations. Therefore, physicians should evaluate whether the estimated values are directly applicable to their patient populations. Third, the data presented in this study are sourced from one randomized clinical trial evaluating patients with RA who continue to have active disease despite prior treatment with MTX, with a relatively small number of patients enrolled to assess the degree of risk with each medication; consequently, the results may not be generalizable to the broader RA population. Fourth, the claims presented in this study regarding the benefit-risk of UPA versus ADA and of UPA versus PBO are limited to the outcomes that were evaluated. Although these outcomes were chosen based on their clinical relevance, the benefit-risk profile could be different for other outcomes.

Certain limitations of clinical trials may have also influenced the results of the present analysis. The trial design for SELECT-COMPARE 
included rescue protocols and trial arm crossover during the trial period. To mitigate the impact of treatment switching, non-responder imputation methods were applied to efficacy results at all time points for patients who failed to respond to treatment or who switched treatment per trial protocol. Safety results were censored at the time of treatment switch for patients who discontinued their randomized treatment. Additionally, SAEs of interest evaluated are generally rare events of low incidence, and as such, randomized clinical trials may not be statistically powered to detect generalizable risks. Future analyses should be conducted to evaluate the therapeutic profile of treatments in other patient populations. Furthermore, clinical trials are conducted under rigorous protocols that may not accurately capture real-world practices, such as the level of patients' compliance to treatment, which are important determinants of real-world treatment effectiveness and tolerability.

\section{CONCLUSIONS}

The present analysis evaluated important efficacy and safety outcomes in patients with RA using NNT and NNH methodology to provide a more clinically meaningful perspective on the benefit-risk comparison between UPA and ADA over 3 years. Our results indicate that UPA consistently showed greater efficacy compared to ADA as evidenced by NNT values $<10$ for the achievement of clinical remission and low disease activity over 26 weeks. This advantage of UPA persisted over the long term, as evidenced by low NNT values $<10$ in comparisons between UPA and ADA over 48 and 156 weeks of treatment for DAS28-CRP $<2.6$. There were limited additional safety risks associated with UPA when compared to ADA, as exemplified by large (and often negative) NNH values and nonsignificant confidence intervals. When the benefit and risk were evaluated together in a hypothetical cohort of 100 patients, our study estimated a greater number of additional patients who would benefit from UPA treatment in comparison with ADA, while, with the exception of herpes zoster, all other AESIs had similar risks between UPA and ADA. Taken together, this analysis of evidence from the SELECT-COMPARE trial suggests a favorable benefit-risk profile of UPA compared to ADA for patients with moderate-to-severe RA who have responded inadequately to MTX.

\section{ACKNOWLEDGEMENTS}

Funding. Sponsorship for this study (NCT02629159) and the journal's Rapid Service Fee were funded by AbbVie, Inc., North Chicago, Illinois, USA. AbbVie participated in the trial design, research, analysis, data collection, interpretation of data, and review and approval of this publication.

Authorship. All named authors meet the International Committee of Medical Journal Editors (ICMJE) criteria for authorship for this article, take responsibility for the integrity of the work as a whole, and have given their approval for this version to be published. All authors had access to relevant data and participated in the drafting, review, and approval of this publication. No honoraria or payments were made for authorship.

Author Contributions. All authors contributed to study conception and design and interpretation of the data. Keith A. Betts, Jayeshkumar Patel, and Rochelle Sun contributed to the collection and assembly of the data, as well as data analysis and interpretation.

Medical Writing, Editorial, and Other Assistance. Medical writing assistance was provided by Mona Lisa Chanda, PhD, an employee of Analysis Group, Inc. The authors thank Ruta Sawant for her assistance with analysis and preparation of the manuscript. Support for this assistance was provided by AbbVie, Inc.

Disclosures. Keith A. Betts and Rochelle Sun are employees of Analysis Group, Inc., which has received consulting fees from the sponsor. Philip Conaghan has been a consultant or 
speaker for AbbVie, BMS, Eli Lilly, Gilead, GSK, Novartis, Pfizer, AstraZeneca, and Galapagos. Stanley Cohen has received consulting fees and research grants from AbbVie, Amgen, Boehringer Ingelheim, Gilead, Pfizer, Roche, and Sandoz. Gerd Burmester has received speaking/consulting fees from AbbVie, Lilly, Galapagos, Janssen, MSD, Pfizer, Roche, and UCB. Eduardo Mysler has received consulting fees and received grants/support from AbbVie, AstraZeneca, BMS, Lilly, GSK, Janssen, Pfizer, Roche, and Sandoz. Peter Nash has received research funding and honoraria for advice and lectures from AbbVie, BMS, Celgene, Lilly, Gilead/Galapagos, Janssen, MSD, Novartis, Pfizer, Roche, Samsung, Sanofi, and UCB. Yoshiya Tanaka has received speaking fees and/or honoraria from Gilead, AbbVie, Behringer-Ingelheim, Eli Lilly, Mitsubishi-Tanabe, Chugai, Amgen, YL Biologics, Eisai, Astellas, BristolMyers, and Astra-Zeneca, and has received research grants from Asahi-Kasei, AbbVie, Chugai, Mitsubishi-Tanabe, Eisai, Takeda, Corrona, Daiichi-Sankyo, Kowa, and Boehringer-Ingelheim. William Rigby has received consulting fees and clinical trial support from AbbVie. Roy Fleischmann has received consulting fees from AbbVie, Amgen, BMS, Galvani, Gilead, GSK, Janssen, Eli Lilly, Novartis, Pfizer, and UCB, and clinical trial grants from AbbVie, Amgen, AstraZeneca, BMS, Flexion, Galvani, Gilead, GSK, Janssen, Eli Lilly, Novartis, Noven, Pfizer, Samumed, Selecta, Teva, UCB, Viela, and Vorso. Jayeshkumar Patel, Tim Shaw, Pankaj Patel, and Jianzhong Liu are AbbVie employees and may own AbbVie stock/stock options.

Compliance with Ethics Guidelines. The SELECT-COMPARE study was conducted per the International Conference on Harmonisation guidelines, applicable regulations, and the Declaration of Helsinki. Study-related documents were approved by the U.S. Central Institutional Review Board (Quorum \#31,009) and other local institutional ethics committees and review boards. All patient data used in this analysis was statistically de-identified and certified as fully compliant with US patient confidentiality requirements outlined in the Health Insurance Portability and Accountability Act of
1996. Because this study relied exclusively on de-identified patient records and did not involve the collection, use, or dissemination of individually-identifiable data, institutional review board approval was not necessary.

Data Availability. AbbVie is committed to responsible data sharing regarding the clinical trials we sponsor. This includes access to anonymized, individual, and trial-level data (analysis datasets), and other information (e.g., protocols and clinical study reports), as long as the trials are not part of an ongoing or planned regulatory submission. This includes requests for clinical trial data for unlicensed products and indications. This clinical trial data can be requested by any qualified researchers who engage in rigorous, independent scientific research, and will be provided following review and approval of a research proposal and statistical analysis plan and execution of a data sharing agreement. Data requests can be submitted at any time and the data will be accessible for 12 months, with possible extensions considered. For more information on the data sharing process, or to submit a request, see https://www.abbvie.com/our-science/clinicaltrials/clinical-trials-data-and-informationsharing/data-and-information-sharing-withqualified-researchers.html

Open Access. This article is licensed under a Creative Commons Attribution-NonCommercial 4.0 International License, which permits any non-commercial use, sharing, adaptation, distribution and reproduction in any medium or format, as long as you give appropriate credit to the original author(s) and the source, provide a link to the Creative Commons licence, and indicate if changes were made. The images or other third party material in this article are included in the article's Creative Commons licence, unless indicated otherwise in a credit line to the material. If material is not included in the article's Creative Commons licence and your intended use is not permitted by statutory regulation or exceeds the permitted use, you will need to obtain permission directly from the copyright holder. To view a copy of this licence, 
visit http://creativecommons.org/licenses/bync/4.0/.

\section{REFERENCES}

1. Radner H, Alasti F, Smolen JS, Aletaha D. Physical function continues to improve when clinical remission is sustained in rheumatoid arthritis patients. Arthritis Res Ther. 2015;17(1):203.

2. Aletaha D, Smolen JS. Diagnosis and management of rheumatoid arthritis: a review. JAMA. 2018;320(13):1360-72.

3. Krishnan E, Lingala B, Bruce B, Fries JF. Disability in rheumatoid arthritis in the era of biological treatments. Ann Rheum Dis. 2012;71(2):213.

4. Verstappen SMM. Rheumatoid arthritis and work: the impact of rheumatoid arthritis on absenteeism and presenteeism. Best Pract Res Clin Rheumatol. 2015;29(3):495-511.

5. Taylor PC, Moore A, Vasilescu R, Alvir J, Tarallo M. A structured literature review of the burden of illness and unmet needs in patients with rheumatoid arthritis: a current perspective. Rheumatol Int. 2016;36(5):685-95.

6. Kohler BM, Gunther J, Kaudewitz D, Lorenz HM. Current therapeutic options in the treatment of rheumatoid arthritis. J Clin Med. 2019;8(7):938.

7. Kiely PD, Brown AK, Edwards CJ, et al. Contemporary treatment principles for early rheumatoid arthritis: a consensus statement. Rheumatology (Oxford). 2009;48(7):765-72.

8. Emery P. Evidence supporting the benefit of early intervention in rheumatoid arthritis. J Rheumatol Suppl. 2002;66:3-8.

9. Gunasekera WM, Kirwan JR. Rheumatoid arthritis: previously untreated early disease. BMJ Clin Evid. 2016;2016:1124.

10. Fraenkel L, Bathon JM, England BR, et al. 2021 American College of Rheumatology guideline for the treatment of rheumatoid arthritis. Arthritis Care Res (Hoboken). 2021;73(7):924-39.

11. Upchurch KS, Kay J. Evolution of treatment for rheumatoid arthritis. Rheumatology (Oxford). 2012;51(Suppl 6):vi28-36.

12. Lin Y-J, Anzaghe M, Schülke S. Update on the pathomechanism, diagnosis, and treatment options for rheumatoid arthritis. Cells. 2020;9(4):880.
13. Yu C, Jin S, Wang Y, et al. Remission rate and predictors of remission in patients with rheumatoid arthritis under treat-to-target strategy in real-world studies: a systematic review and meta-analysis. Clin Rheumatol. 2019;38(3):727-38.

14. Pope J, Sawant R, Tundia N, et al. Comparative efficacy of JAK inhibitors for moderate-to-severe rheumatoid arthritis: a network meta-analysis. Adv Ther. 2020;37(5):2356-72.

15. Ogata A, Kato Y, Higa S, Yoshizaki K. IL-6 inhibitor for the treatment of rheumatoid arthritis: a comprehensive review. Mod Rheumatol. 2019;29(2): 258-67.

16. Virtanen AT, Haikarainen T, Raivola J, Silvennoinen O. Selective JAKinibs: prospects in inflammatory and autoimmune diseases. BioDrugs. 2019;33(1):15-32.

17. Morrissey H, Ball PA, Askari A, Nouri AK. Janus kinase enzyme (JAK) inhibitors and rheumatoid arthritis: a review of the literature. Int J Curr Pharm Res. 2019;11(6). http://dx.doi.org/10.22159/ijcpr. 2019v11i6.36343.

18. Kremer JM, Bingham CO III, Cappelli LC, et al. Postapproval comparative safety study of tofacitinib and biological disease-modifying antirheumatic drugs: 5-year results from a united statesbased rheumatoid arthritis registry. ACR Open Rheumatology. 2021;3(3):173-84.

19. Winthrop KL. The emerging safety profile of JAK inhibitors in rheumatic disease. Nat Rev Rheumatol. $2017 ; 13(4): 234-43$.

20. Curtis JR, Lee EB, Kaplan IV, et al. Tofacitinib, an oral Janus kinase inhibitor: analysis of malignancies across the rheumatoid arthritis clinical development programme. Ann Rheum Dis. 2016;75: 831-41.

21. Biggioggero M, Becciolini A, Crotti C, Agape E, Favalli EG. Upadacitinib and filgotinib: the role of JAK1 selective inhibition in the treatment of rheumatoid arthritis. Drugs Context. 2019;8: 212595.

22. Mohamed MF, Beck D, Camp HS, Othman AA. Preferential Inhibition of JAK1 Relative to JAK3 by upadacitinib: exposure-response analyses of ex vivo data from 2 phase 1 clinical trials and comparison to tofacitinib. J Clin Pharmacol. 2020;60(2):188-97.

23. European Medicines Agency. Rinvoq (upadacitinib)-product information. 2019. https://www. ema.europa.eu/en/medicines/human/EPAR/rinvoq. Accessed 1 Sept 2021. 
24. United States Food and Drug Administration. RIN$\mathrm{VOQ}^{\mathrm{TM}}$ (upadacitinib)-Highlights of prescribing information. Label-package Insert. 2019. https:// www.accessdata.fda.gov/drugsatfda_docs/label/ 2019/211675s000lbl.pdf. Accessed 1 Sept 2021.

25. Pharmaceuticals and Medical Devices Agency (PMDA). List of approved products-new drugs: FY 2019. https://www.pmda.go.jp/english/reviewservices/reviews/approved-information/drugs/ 0002.html. Accessed 1 Sept 2021.

26. Burmester GR, Kremer JM, Van den Bosch F, et al. Safety and efficacy of upadacitinib in patients with rheumatoid arthritis and inadequate response to conventional synthetic disease-modifying antirheumatic drugs (SELECT-NEXT): a randomised, double-blind, placebo-controlled phase 3 trial. Lancet. 2018;391(10139):2503-12.

27. Genovese MC, Fleischmann R, Combe B, et al. Safety and efficacy of upadacitinib in patients with active rheumatoid arthritis refractory to biologic disease-modifying anti-rheumatic drugs (SELECTBEYOND): a double-blind, randomised controlled phase 3 trial. Lancet. 2018;391(10139):2513-24.

28. Smolen JS, Pangan AL, Emery P, et al. Upadacitinib as monotherapy in patients with active rheumatoid arthritis and inadequate response to methotrexate (SELECT-MONOTHERAPY): a randomised, placebocontrolled, double-blind phase 3 study. Lancet. 2019;393(10188):2303-11.

29. van Vollenhoven R, Takeuchi T, Pangan AL, et al. Efficacy and safety of upadacitinib monotherapy in methotrexate-naive patients with moderately to severely active rheumatoid arthritis (SELECTEARLY): a randomized, double-blind, active-comparator, multi-center, multi-country trial. Arthritis Rheumatol. 2020;72(10):1607-20.

30. Fleischmann R, Pangan AL, Song $\mathrm{IH}$, et al. Upadacitinib versus placebo or adalimumab in patients with rheumatoid arthritis and an inadequate response to methotrexate: results of a phase III, double-blind, randomized controlled trial. Arthritis Rheumatol. 2019;71(11):1788-800.

31. Fleischmann R, Mysler E, Bessette L, et al. POS0087 long-term safety and efficacy of upadacitinib or adalimumab in patients with rheumatoid arthritis: results at 3 years rrom the SELECT-COMPARE study. Ann Rheum Dis. 2021;80(Suppl 1):251.

32. Citrome L, Ketter TA. When does a difference make a difference? Interpretation of number needed to treat, number needed to harm, and likelihood to be helped or harmed. Int J Clin Pract. 2013;67(5): 407-11.
33. Fleischmann R, Song IH, Enejosa J, et al. Long-term safety and effectiveness of upadacitinib or adalimumab in patients with rheumatoid arthritis: results at 72 weeks from the select-compare study. Ann Rheum Dis. 2020;79(Suppl 1):323.

34. Smolen JS, Aletaha D, Bijlsma JWJ, et al. Treating rheumatoid arthritis to target: recommendations of an international task force. Ann Rheum Dis. 2010;69(4):631.

35. Salaffi F, Ciapetti A. Clinical disease activity assessments in rheumatoid arthritis. Int J Clin Rheumatol. $2013 ; 8(3): 347$.

36. Aletaha D, Nell VP, Stamm T, et al. Acute phase reactants add little to composite disease activity indices for rheumatoid arthritis: validation of a clinical activity score. Arthritis Res Ther. 2005;7(4): R796.

37. Felson D. Defining remission in rheumatoid arthritis. Ann Rheum Dis. 2012;71(0 2):i86-8.

38. Bruce B, Fries JF. The Stanford health assessment questionnaire: dimensions and practical applications. Health Qual Life Outcomes. 2003;1(1):20.

39. Altman DG. Confidence intervals for the number needed to treat. BMJ. 1998;317(7168):1309-12.

40. Laupacis A, Sackett DL, Roberts RS. An assessment of clinically useful measures of the consequences of treatment. N Engl J Med. 1988;318(26):1728-33.

41. Cook RJ, Sackett DL. The number needed to treat: a clinically useful measure of treatment effect. BMJ. 1995;310(6977):452-4.

42. Citrome L. Quantifying risk: the role of absolute and relative measures in interpreting risk of adverse reactions from product labels of antipsychotic medications. Curr Drug Saf. 2009;4(3):229-37.

43. Gopal S, Berwaerts J, Nuamah I, Akhras K, Coppola $D$, Daly E, et al. Number needed to treat and number needed to harm with paliperidone palmitate relative to long-acting haloperidol, bromperidol, and fluphenazine decanoate for treatment of patients with schizophrenia. Neuropsychiatr Dis Treat. 2011;7:93-101.

44. Krishnan E, Tugwell P, Fries JF. Percentile benchmarks in patients with rheumatoid arthritis: health assessment questionnaire as a quality indicator (QI). Arthritis Res Ther. 2004;6(6):R505.

45. Strand V, van Vollenhoven RF, Lee EB,et al. Tofacitinib or adalimumab versus placebo: patient-reported outcomes from a phase 3 study of active rheumatoid arthritis. Rheumatology (Oxford). 2016;55(6):1031-41. 
46. Strand V, Kremer JM, Gruben D, Krishnaswami S, Zwillich SH, Wallenstein GV. Tofacitinib in combination with conventional disease-modifying antirheumatic drugs in patients with active rheumatoid arthritis: patient-reported outcomes from a phase III randomized controlled trial. Arthritis Care Res. 2017;69(4):592-8.

47. European Medicines Agency. Olumiant-baricitinib: medicine overview. 2018. https://www.ema. europa.eu/en/medicines/human/EPAR/olumiant. Accessed 1 Sept 2021.
48. European Medicines Agency. Xeljanz-medicine overview. 2019. https://www.ema.europa.eu/en/ medicines/human/referrals/xeljanz. Accessed 1 Sept 2021.

49. U.S. Food and Drug Administration. OLUMIANT (baricitinib): label-package insert. 2020. https:// www.accessdata.fda.gov/drugsatfda_docs/label/ 2020/207924s002lbl.pdf. Accessed 1 Sept 2021.

50. U.S. Food and Drug Administration. XELJANZ® (tofacitinib)-label-package insert. 2020. https:// www.accessdata.fda.gov/drugsatfda_docs/label/ 2020/203214s026lbl.pdf. Accessed 1 Sept 2021. 